Cahiers de recherches médiévales

\title{
Figures de César dans le Violier des Histoires romaines
}

Denis Hüe

\section{(2) OpenEdition \\ Journals}

Édition électronique

URL : https://journals.openedition.org/crm/855

DOI : $10.4000 / \mathrm{crm} .855$

ISSN : 1955-2424

Éditeur

Honoré Champion

Édition imprimée

Date de publication : 30 mars 2006

Pagination : 149-165

ISSN : 1272-9752

\section{Référence électronique}

Denis Hüe, «Figures de César dans le Violier des Histoires romaines », Cahiers de recherches médiévales [En ligne], 13 spécial | 2006, mis en ligne le 03 avril 2009, consulté le 15 décembre 2022. URL : http:// journals.openedition.org/crm/855 ; DOl : https://doi.org/10.4000/crm.855 


\section{RM}

\section{Figures de César dans le Violier des Histoires romaines}

Du Violier des Histoires romaines, on ne savait, jusqu'à il y a peu, pas grandchose; la seule édition était celle procurée par G. Brunet, dans la "Bibliothèque elzévirienne», en 1858. Une édition récente a tenté de combler l'oubli dans lequel était plongé ce texte, et les recherches bibliographiques récentes ne m'ont indiqué qu'un seul article, de J. Chocheyras, sur les figures féminines dans l'œuvre ${ }^{1}$. Autant dire que ce texte, malgré son grand intérêt, n'a pas été particulièrement étudié. Une des raisons tient peut-être aux difficultés que pose l'édition Brunet, qui annonce le Violier comme une «Ancienne traduction françoise des Gesta Romanorum»: le volume ne correspond que partiellement à cette définition, et a dû décevoir plus d'un lecteur.

On sait que les Gesta Romanorum sont un recueil d'exempla datant de la fin du XIII ${ }^{\mathrm{e}}$ ou du début du XIV $^{\mathrm{e}}$ siècle, rassemblant un nombre variable de récits accompagnés chacun de leur moralisation. Les attributions anciennes, à Hélinant de Froidmont ou à Bersuire, sont aujourd'hui abandonnées, l'un étant sans doute trop ancien, le second trop récent, et le texte reste anonyme, et très largement; l'abondance et la diversité des manuscrits répartis sur l'ensemble du continent vont jusqu'à interdire une détermination d'origine, et il n'est pas possible, en l'état actuel, de déterminer d'où vient ce recueil, très abondamment représenté - mais le plus souvent dans des versions distinctes - sur chacune des régions et dans chacune des langues européennes. Ainsi, dans la version latine, qui semble se fixer sur le plan éditorial autour de 1480 - la tradition manuscrite est plus complexe encore, en ce qu'elle est difficile à dater - on compte généralement 181 histoires, alors que notre version, la version française que constitue le Violier est composée de 149 contes, repris en revanche, et sans exception, du texte latin.

Le titre même de la version, Gesta Romanorum, ne doit pas cependant faire illusion. Si l'ambition est de proposer des exemples édifiants, ils participent d'une vision pour le moins anhistorique de l'empire romain. Rien que pour notre sujet, Jules César, on le voit promulguer des lois qu'il n'a jamais édictées, et une statue de lui semble avoir été édifiée dans les vingt ans qui suivent la fondation de Rome. Pas d'histoire donc, ou du moins pas au sens où l'entendent les historiens d'aujourd'hui. Les références à l'antiquité sont rarement appuyées sur les auteurs que l'on s'attendrait à y voir. Mieux, des références à Paul le Lombard (Paul Diacre) ou à

\footnotetext{
${ }^{1}$ L'édition du Violier des Histoires Romaines, ancienne traduction française des Gesta Romanorum, nouvelle édition, revue et annotée par M. G. Brunet, Paris, Jannet «Bibliothèque elzévirienne », 1858, est maintenant disponible sur le site Gallica de la Bibliothèque nationale de France. L'édition récente est établie par Geoffroy Hope, Droz, «Textes Littéraires Français », 2002. On pourra également consulter sur le site Gallica le fichier PDF de l'édition Denis Janot, 1529. Quant à l'article de J. Chocheyras, «Le masculin et le feminin dans un recueil de moralia, Le Violier des Histoires Romaines (1521)», Recherches et travauxUniversite de Grenoble, U.E.R. de lettres, Année : 1996, N 50, p. 151-155.
}

Cahiers de Recherches Médiévales, 13spé, 2005 
Gervais de Tilbury nous montrent que nous ne sommes pas dans une perspective propre à l'Antiquité. Le volume contient de plus des histoires assez développées, qu'il s'agisse de la légende de saint Eustache ou de celle de Grégoire Pape, celle de saint Alexis ou celle d'Apollonius de Tyr.

Pourquoi, demandera-t-on, aborder une œuvre où la figure de César semble aussi absente, alors qu'il est le sujet même de nos préoccupations? Le lecteur moderne est pénétré d'une vision qu'il s'imagine fiable de l'Antiquité gréco-latine; pour lui, Jules César correspond à une donnée historique ; entre XIII ${ }^{\mathrm{e}}$ et $\mathrm{XVI}^{\mathrm{e}}$ siècle, nous sommes à un moment où s'opère, et sur plusieurs périodes, une cristallisation d'une Antiquité imaginaire; c'est donc le Jules César des chroniques et des poètes tel qu'il est reçu au Moyen Âge qui nous intéresse, mais aussi celui des moralistes du XIII ${ }^{\mathrm{e}}$ siècle, celui enfin de la traduction qui est faite au début du XVI ${ }^{\mathrm{e}}$ siècle. Pour en relever les traces, on travaillera dans un premier temps sur le recueil du Violier lui-même et sur les enjeux qu'il tente de relever; une étude des occurrences de Jules César dans le recueil permettra de mieux comprendre le statut non seulement de la romanité, mais aussi des figures historiques et de leur utilisation au cours du Moyen Âge. On pourra enfin s'attarder sur l'épisode bien connu du passage du Rubicon et ses diverses variantes textuelles, qui permettront de dégager quelques-uns des procédés d'appropriation de la matière antique.

Dans ce genre de recueil, autant il est difficile d'établir, par des moyens philologiques, un historique des versions et une hypothétique filiation des textes, autant il est vain de chercher le détail des sources. Quelques références à Pline ou saint Augustin peuvent prétendre à garantir une autorité, mais elles sont souvent imprécises, et ne sauraient constituer une source au sens précis du terme. Des mots, de surcroît, invitent à rechercher une source orientale à certains de ces récits, et on les retrouve effectivement dans des recueils comme le Kalila et Dymna. Ce qui nous retiendra ici, en quelque sorte, ce ne peut être la dimension strictement textuelle des récits, quasiment fortuite et issue d'une tradition textuelle extrêmement complexe.

C'est, plutôt, la capacité de proposer une moralisation détaillée sur le sujet: ce que nous voyons apparaitre ainsi, c'est la démarche de la moralisation, qui touche son sommet au XIV siècle, dans la capacité à décomposer un récit pour le réduire à des éléments signifiants dont on va explorer la signification. La force de cette démarche est d'être à la fois envahissante et presque automatique, et de n'être en même temps jamais exclusive. Le sens d'un récit, d'un détail, d'un mot, ne naît pas seulement de lui-même, dans une sorte d'univocité heureuse; il surgit en quelque sorte de l'entourage, qui va lui donner son inflexion propre. Qui a lu les œuvres de Bersuire se souvient des balancements vel dic qui scandent les démarches de moralisation : un objet signifie une chose et son contraire, comme s'il était présent dans le discours en valeur absolue. C'est ce qui fait que bien des recueils d'exempla se contentent d'un titre ou d'une rubrique pour orienter l'interprétation. Étienne de Bourbon, par exemple, ne dit rien généralement dans son Tractatus de diversis materiis praedicabilibus de la signification de ce qu'il rapporte; ou plutôt il l'inclut dans une sorte de discours et de propos général qui infléchit le sens qu'il veut en donner. Il n'en est pas même ainsi dans les Gesta, dont n'émerge aucun projet parénétique précis, et pas en tous cas un désir comparable de faire somme. 
149 contes pour le Violier des histoires romaines contre plus de 180 dans les Gesta : cette version est donc plus brève, et le projet général, s'il y en a un, encore moins perceptible; on ne connaît pas de version manuscrite du Violier, mais on sait en revanche que plusieurs versions imprimées existent, dont certaines illustrées, comme celle de Denis Janot en 1529.

La première édition, publiée en 1521 par Jehan de La Garde comporte une préface à Louise de Savoie, préface dans laquelle l'auteur de la traduction présente «ce livre si mal digeste, compillé et traduit », et souligne les vertus de la dame :

qui en vous sont infuses si habondantement que toutes autres transpassent et excedent, tant soit la fleur de leur honneur et gloire pullulante sus le germe de l'accroissement de tous incomparez merites, tout ainsi que le verd therebinte plus amplement sur tous arbres ses branches et rainceaulx dilate. ${ }^{2}$

La métaphore végétale qui gouverne le propos dédicatoire se retrouvera en effet dans le titre :

Ce livre donc accepterez nommé le Violer des hystoires romaines, à cause qu'il contient maintes gestes et propos divers des faictz des romains, qui moult sont plaisantes et delectables, et encore plus prouffitables à cause des sens moraulx spirituelz desquels elles sont fructueusement revestues pour le nouvel parement, edification et interieure beaulté de toutes bonnes meurs et conditions, et tant que toute vertus se peult dedans considerer et mirer [...] a l'introduction de tous lecteurs, comprehencion de bonnes meurs, refloriture de frais memoire. ${ }^{3}$

Le mot de violier peut renvoyer à plusieurs choses différentes; d'une part le lieu planté de violettes, et on dit alors un violier comme on dit une roseraie ; " on appelle aussi violier la giroflée des jardins, cheiranthus incanus, L.»(Littré); certains dictionnaires présentent le giroflier comme la plante qui porte la giroflée, au même titre que le rosier porte la rose. Ce jeu de synonymie nous permet de comprendre non seulement le violier comme une plante portant violette ou giroflée, mais aussi comme équivalent exact du giroflier. Ce mot renvoie on le sait non seulement au nom même de la plante, mais aussi à la petite boîte précieuse que les aristocrates portaient avec eux, remplis de clous de girofle, pour parfumer leur haleine.

Le violier, comme le giroflier, devient une sorte de drageoir aux épices, petit objet destiné à receler des trésors, et associé aux fleurs, par les parfums qu'il recèle : l'allusion au térébinthe qui courait au début de l'épître dédicatoire annonçait cette métaphore, et permet aussi de reprendre l'allusion évidente au florilège, à l'anthologie, à l'excellence du choix qui est offert. Cette anthologie, pour finir, est adressée à la «florissant memoire ${ }^{4}$ » de la princesse. Tout se passe comme si chaque allusion florale constituait une sorte de signal soulignant un élément essentiel du propos du traducteur; ici, la mémoire renvoie au registre de la merveille, de

${ }^{2}$ Éd. Hope, p. 7

${ }^{3}$ Ibid.

${ }^{4}$ Id., p. 8. 
l'anecdote, de la chose mémorable par sa curiosité ou l'enseignement que l'on peut en tirer. Inutile de rappeler combien, au-delà même du genre de l'exemplum, le Moyen Âge et la Renaissance sont friands de ce genre de récits. Mentionnons juste, pour encadrer le propos, les Otia imperialia de Gervais de Tilbury, qui constitueront significativement une des sources importantes de l'œuvre, et quelques années plus tard, les Histoires prodigieuses de Boaistuau.

Mais, par rapport aux merveilles rapportées par Gervais, le commentateur ajoute un élément essentiel, l'annonce des «des sens moraulx spirituelz desquels elles sont fructueusement revestues pour le nouvel parement, edification et interieure beaulté de toutes bonnes meurs et conditions »: l'adjonction de moralisation n'est pas seulement l'ajout d'un sens moral, mais réellement un habit qui, au lieu d'être «floral» comme l'ont été bien des descriptions des récits, devient cette fois-ci fructueux.

Élément supplémentaire, le passage de l'extériorité - le sens moral et spirituel revêt ces histoires - vers une intériorité, l'interieure beaulté dont elle va parer la dame. Les images populaires diffusaient alors les représentations de Fortunatus et de sa bourse, dont l'intérieur et l'extérieur sont identiques en sorte que qui la possède possède le monde. Ici, c'est le divertissement qui, bien compris, apporte un embellissement intérieur.

La forme et l'ambition proposées par le violier sont donc d'une autre nature que celle qui dirigeaient les Gesta Romanorum, en ce qu'elles ne s'adressent pas au prédicateur, mais à un lectorat privé, ciblé, au travers de la figure tutélaire de Louise de Savoie, comme étant à la fois aristocratique et féminin. On pourrait souligner enfin combien la mère du roi de France est fréquemment dédicataire de ces traductions françaises qui fleurissent au début du XVI $\mathrm{XI}^{\mathrm{e}}$ siècle, comme si elle était emblématique de cette curiosité de l'aristocratie pour de nouvelles formes d'œuvres morales. J'ai évoqué ailleurs le manuscrit de la traduction du Miroir des Dames par Isambert de Saint-Léger ${ }^{5}$, qui joue sur des procédures et des captationes comparables.

Public aristocratique que celui du Violier? Il s'agit évidemment plus d'un vœu pieux que d'une réalité ; tout ce que l'on peut dire, c'est qu'il ne nous reste aucun manuscrit de ce texte, et que l'on peut même se demander s'il a existé un exemplaire de dédicace. Le privilège qui est imprimé au début de la première édition présente «Jehan de la garde, libraire juré en l'université de Paris, conteant que pour le proufit, utilisté de plusieurs personnes, il avait fait translater par homme expert et litteré les gestes romaines moralisées " ${ }^{6}$; si l'on ajoute à une telle affirmation le souci clairement formulé de la crainte du préjudice que pourrait entraîner une contrefaçon, le doute n'est plus permis: la traduction est originale, destinée à l'imprimerie, et censée recevoir un accueil favorable. Le succès escompté est probable, puisque l'on voit paraître un ensemble d'éditions relevées par Brunet: deux éditions de P. Le Noir, une sans date, une de 1525, et enfin une édition de Denis Janot, en 1529: quatre éditions en une petite dizaine d'années, voilà qui

5 D. Hüe, «Un Miroir des dames à la Renaissance», Miroirs et jeux de miroirs dans la littérature médiévale, sous la direction de F. Pomel, P.U. Rennes 2, «Interférences», 2003, p. 63-77.

Éd. Hope, p. 5. 
témoigne d'un réel succès de l'œuvre. On voit que la dimension royale attachée symboliquement à l'œuvre cherche à la rattacher à la tradition des traités parénétiques et didactiques, miroirs des Princes ou Secret des secrets, qui utilisent la figure du souverain pour, équivalence microcosme-macrocosme oblige, dispenser un savoir général valable pour chacun. Faux miroir des Princes, le Violier semble viser un public populaire, joue sur la noblesse de ses propos, et sur le luxe supposé de l'édition, qui est manifesté par l'illustration.

Ces éditions sont en effet toutes illustrées, celle de J. de la Garde, les deux éditions de H. le Noir, et celle enfin de D. Janot, qui a manifestement repris les mêmes bois. On reconnaît ainsi, au f I de l'édition de 1529, la même illustration qu'au f II de l'édition originale.

Ils proviennent, Hope le confirme, d'une édition encore antérieure des Fantasies de la mere sote. Je ne comprends pas pourquoi Hope affirme que la série de bois proposée par l'édition Janot est différente de la première édition: on retrouve un certain nombre de bois, tout à fait identiques à l'usure près qu'expliquent aisément les huit années d'écart, dans les deux éditions. Il faut en conclure que les familles de libraires, qui étaient souvent associées, ont pu se prêter ou se louer les bois pour des campagnes de publication comme celle-ci. Certains bois, comme celui qui représente la scène de présentation du volume, sont évidemment différents ; chaque libraire a en effet des bois tout prêts pour des scènes de ce genre et l'on peut penser qu'il n'allait pas louer, à prix d'argent, des bois dont il pouvait se passer.

Après cette rapide présentation du Violier, on comprend mieux je crois les enjeux qui l'entourent: il s'agit d'une version française d'un recueil très connu des prédicateurs, transposé dans notre langue afin de rencontrer un nouveau public, par un libraire qui pense «faire un coup». le public, c'est celui d'une petite aristocratie, d'une petite bourgeoisie, celui qui pouvait être séduit par les homélies des Prêcheurs et par leur talent à utiliser des histoires, à la fois désireux de divertissement et d'édification. Livre précieux par son contenu, et qui s'efforce d'avoir une certaine tenue éditoriale, il est en même temps de souffle et d'ambitions un peu courtes : c'est le merveilleux du conteur qui domine plus qu'un réel savoir, c'est l'étrangeté de l'anecdote qui va primer sur sa fiabilité historique. Il est à noter cependant que, si les récits commencent à peu près tous de la même façon, ils n'insistent pas systématiquement sur l'autorité qui garantit l'anecdote; certes, on rencontre quelques références à saint Augustin, à Sénèque, à Valère Maxime. Mais il est révélateur que les références précises sont presque toujours absentes et, quand elles sont présentes, fausses. Dans la liste des autorités, on pourra ainsi relever 5 références à Valère Maxime, 4 à Pline, 3 à saint Augustin, 2 à Sénèque, et Gervais de Tilbury, 1 à Ovide, Macrobe, Flavius Josephe, Eusèbe de Césarée, Cicéron. Si ces références sont attendues, ont peut s'étonner en revanche de trouver une référence à la Vie des Pères (134), qui n'a rien d'antique, et plus étonnants encore, deux renvois aux Gesta romanorum : le paradoxe ne vient pas de la traduction, mais du texte latin, qui spécifie Legitur in gestis romanorum (cap. 34), comme s'il existait une sorte d'entité faits des Romains avant même la rédaction des recueils d'exempla. L'essentiel n'est pas dans la source, on le voit; il est dans la garantie d'authenticité, dans la caution qu'un nom est censé donner: nous sommes ici dans l'apologétique, 
dans l'homilétique et pas dans l'historique. À preuve, les chapitres n'ont pas pour titre le sujet de l'anecdote, mais l'objet de la moralisation: «De l'estat tripple de ce monde » (ch. 85), «De la vraye probation d'amytié » (ch. 109), «De la mort de Christ innocente (ch. 112).

L'historicité comme l'autorité n'ont donc pas de valeur propre, et ont une simple fonction d'effet de réel: si l'enseignement qui en est issu est aussi véridique, il est évident que l'histoire qui les suscite l'est aussi, à quelque titre que ce soit. C'est la raison pour laquelle de très nombreux chapitres auront pour simple indication «aucun empereur» (5 fois) «Aucun noble personnage», 1 fois ; «Aucun prince», 1 fois, «aucun roi », 5 fois. Cela reprend fidèlement les caractéristiques établies dès le texte latin des Gesta romanorum :

"Quidam imperator erat, qui...": so oder ähnlich beginnen in dem Geschichtenbuch, das sie unter dem Titel „Gesta romanorum“ in späteren Mittelalter im Einflußbereich der lateinischen Sprache einer denkbar großen Beliebtheit und weiten Verbreitung erfreuen konnte, ein beträchtlicher Teil der Erzählungen. Allerdings brauchte nicht immer gleich ein Kaiser am Eingang einer Geschichte zu stehen, sondern häufig genügte schon ein Köning: ,erat quidam rex qui... “7

Dans le Violier, de simples mentions comme «il estoit», ou «jadis», ou «jadis estoit» finissent par représenter 10 occurrences, éventuellement associées en «jadis un roy»; on voit apparaître «quelque empereur, quelque roy» (une fois chacun), puis des mentions comme «ung chevalier» (4 fois), «ung empereur» (6 fois), mais surtout « ung roy (19 fois)

Pour qui connaît un minimum d'histoire romaine, une telle proportion prouve, avant toute lecture, la part de légende qui est ici recueillie, et c'est de cela qu'il est question évidemment.

Mais, dans une démarche qui est censée asseoir l'authenticité des anecdotes, ce n'est pas tant le fait que des choses aient eu lieu qu'elles se soient déroulées en un temps ou un moment précis. Les stratégies narratives s'efforcent de placer des noms propres exemplaires et prestigieux comme garants des fables rapportées, ne serait-ce que parce qu'elles sont contemporaines, et parfois parce que les cautions historiques en sont les héros. Ainsi, Vespasien apparaît 2 fois, comme Maximien et Dioclétien ; Titus 3, Tibère 3, Pompée 3, César 3 fois, mais Alexandre 9 fois. Si César ne figure pas ici comme un personnage central de l'histoire antique, il est mis au même rang que des empereurs dont la tradition médiévale ne dit pas grand-chose. On peut ajouter que, dans la tradition textuelle, le mot Caesar ne renvoie pas forcément à Jules César, mais devient une sorte d'équivalent du titre d'empereur qu'il ne porta jamais, d'où les noms, on le sait, de Tsar ou de Kaiser. Néanmoins, on peut admettre que nos textes, qui connaissent les mots imperator et rex, utilisent le nom de César comme un nom propre plus que comme un titre.

Ces trois occurrences ne sont certes pas très abondantes, surtout si on les rapproche des mentions d'Alexandre, bien plus fréquentes. Mais, rapportées à ce que propose par exemple le traité d'Étienne de Bourbon, qui présente 16 occurrences de

${ }^{7}$ Johannes Schneider, «Das Fortleben der römischen Kaiser in den Gesta romanorum », Klio Berlin, Akademie-Verlag, 52, 1970, p. 395-410 
César sur 2857 exempla, soit environ $0,5 \%{ }^{8}$, les $2 \%$ que représentent les mentions de César dans l'ensemble de l'œuvre ne sont pas négligeables. Rapportées aux noms d'empereurs romains présents dans le texte, c'est environ $8 \%$ des récits qui concernent César. On peut conclure que le personnage, s'il n'est pas le premier dans les traditions légendaires qui courent tout au long du Moyen Âge, n'est pas non plus oublié.

Avant de travailler sur les récits eux-mêmes, il suffira de rappeler combien ils sont indépendants de toute historicité ; et si quelques-uns des signes annonciateurs de la mort de César figurent chez Suétone, c'est presque de façon fortuite. Le rappel du premier chapitre dans lequel figure le nom de César permettra de cerner quelques-unes des caractéristiques du rôle qu'on lui fera jouer.

La première occurrence de la figure de César qui nous retiendra le montre comme tout à fait anecdotique à l'histoire rapportée :

90 De la virille bataille de Jesuchrist et de sa victoire

Cesar regna, au royaulme duquel estoit ung noble chevalier et fort qui chevauchoit une fois par une forest où il veit ung crapault et ung serpent qui ensemblement combatoient. Mais le crapault prevalut et eut victoire. Ce voyant, le chevalier ayda au serpent et blessa fort le crapault. Toutesfois, le chevalier fut griefvement blessé par le crapault. ${ }^{9}$

Le récit se poursuit dans la marque de la fidélité du serpent, qui va sucer la plaie infectée du chevalier jusqu'à sa guérison, le crapaud étant ensuite tué par les serviteurs du chevalier. On retrouve une série de motifs traditionnels proches du combat d'Yvain, du lion et du serpent, ce dernier toutefois ayant un rôle positif qui ne lui est pas exceptionnel, on se souvient que cet animal est un des attributs de la Prudence. L'interprétation morale de ce conte est assez complexe :

Le chevalier est nostre seigneur Jesuchrist. Le crapault est le dyable d'enfer et le serpent, l'homme. L'homme peult estre dict serpent pour deux causes. Pour le venin de peché et pour la bataille qu'il doit avoir contre le dyable. ${ }^{10}$

Le moralisateur rejoue ainsi la chute et le salut, la seule originalité du serpent étant justement dans son ambivalence - les encyclopédistes n'attribuaient-ils pas à cet animal la capacité d'absorber le venin de la terre qu'ils habitaient, la purifiant ainsi ? le plus étonnant reste cependant l'interprétation qui est faite des deux premiers mots du texte: «Cest empereur est le pere celeste». Toute figure royale représente Dieu dans les Gesta, de même qu'elle se veut représentante de Dieu dans son idéologie politique.

${ }^{8}$ Cf. J. Berlioz, «Héros païen et prédication chrétienne : Jules César dans le recueil d'exempla $\mathrm{du}$ dominicain Etienne de Bourbon (mort vers 1261)», dans Exemplum et Similitudo. Alexander the Great and other heroes as points of reference in medieval literature, ed. by W. J. Aerts and M. Gosman. Actes du Colloque, Groeningen, 14-16 mai 1986, Groeningen: Egbert Forster, 1988 (Mediaevalia Groningana, 8), p. 123-143. Merci à B. Méniel de m'avoir signalé cette référence.

9 Op. cit., p. 256.

${ }^{10}$ P. 256. 
Ainsi César est-il une figure divine, privilège qu'il partage cependant avec presque chaque figure impériale du Violier.

Le chapitre 4 , «De la justice et ordonnance de Cesar », évoque une règle qu'il aurait instaurée, telle que «si aucun ravissoit et prenoit femme par force qu'il fust en la juridicion des femmes soubmys pour sçavoir se il mourroit ou se il la prendroit en mariaige sans douaire. Le cas advint que aucun de nuyt ravit deux femmes ensemblement desquelles l'une demanda la mort du capteur et l'autre sa delivrance par mariaige ». De là, un court débat fait apparaître que celle qui demande la peine la plus clémente sera entendue, et la «moralisation sur le propos » nous montre qu'il s'agit d'une représentation de Justice et de Miséricorde :

Cest empereur est nostre seigneur Jesuchrist. Le ravisseur est chascun pecheur qui ravist et violle deux femmes, c'est assavoir justice pareillement misericorde, qui toutes deux sont filles de Dieu. Le ravisseur est devant le divin tribunal convocqué quant l'ame par mort est du corps separée. La premiere, qui est justice, contre le transgresseur instantement allegue que il doit mourir de mort eternelle selon la loy de justice. Mais l' autre, qui est misericorde divine, tout à l'opposite dit que par le sacrement de penitence doit le pecheur avoir pardon et estre joinct à elle, tellement que par cela est le pecheur à la grace de Dieu assemblé. ${ }^{11}$

Il y a peu de chances qu'une telle histoire soit attestée chez les chroniqueurs antiques, et l'on voit combien elle relève de la tradition homilétique; un législateur et garant de la loi - établit une règle qui se trouve impossible à appliquer, et justice et miséricorde s'affrontent sur la conduite à tenir. Ici, c'est en filigrane un procès de paradis qui se tient, reconnaissable pour tout lecteur de la fin du Moyen Âge, tant la figure en est fréquente, dans la littérature comme dans la société. Ce qui retiendra peut-être ici, c'est en fait la fonction juridique qui est attachée à Jules César, fonction qui est mise en place tout au long du dispositif «Cesar regna à Romme lequel ordonna en son temps » est suivi de «En fin le juge leur conceda», et enfin de «Cest empereur est nostre seigneur Jesuchrist»: la figure de l'empereur n'est pas celle d'un général, mais celle du législateur, attachée au fondement du droit romain, au point qu'en France on ne pouvait étudier à Paris un droit où constamment la caution suprême était l'empereur et non le roi. L'empire d'occident jouait par ailleurs sur le statut quasiment divin du souverain, et le décorum d'Aix-la-Chapelle comme le manteau du sacre de Bamberg en sont de bons exemples. À ce titre, comme fondateur de l'empire et garant des lois, Jules César est directement à même d'endosser un rôle de sage qui est à la fois laïc et représentant de la justice divine. Mais César n'est législateur, somme toute, que parce qu'il est figure exemplaire de la justice, figure suscitée pour l'occasion.

Une troisième occurrence de la figure de Jules César se trouvera dans le récit 90, qu'il importe peut-être de citer intégralement, tant il semble riche d'enseignements :

${ }^{11}$ Éd. Hope, p. 18-19. 
89 De la mort

On lict es cronicques que l'an vingt et deuxiesme de la fondation de Romme, les Rommains firent eriger une columne de marbre dedans le capitolle de la cité. Et sus la columne mirent l'imaige de Julius Cesar, et sus l'ymaige son nom escript. Mais celuy Cesar eut trois signes merveilleux devant que mourir. Le centiesme jour devant sa mort, la fouldre tomba devant son ymaige, rasant de son nom suscript la premiere lettre. La nuyt de sa mort precedente, les fenestres de sa chambre furent si impetueusement ouvertes qu'il estimoit que la maison tombast. Le mesme jour qu'il fut tué comme il entroit au Capitolle, baillées luy furent des lettres indices et qui luy demonstroient sa mort, lesquelles s'il les eust leues, il fust evadé de son occision et meurtre.

L'exposicion sus le propos

Ainsi est il de nous et de Dieu. Dieu nous fait trois signes pour la mort eternelle fuyr et eviter. Le premier, l'effacement et l'abolition de la premiere lettre du nom, qui signifie de richesse habondance. Ce sont les richesses qui font avoir nom en la terre. Jamais homme n'est prisé en honneur s'il n'a des biens. Si on veult preferer quelq'ung, il convient qu'il soit non pas bon, juste, sainct, mais riche ${ }^{12}$. Pour tant, quant Dieu veult les riches saulver, il oste la premiere lettre de son nom, qui sont les richesses qui causent dampnation le plus souvent. Le second signe de Julius fut que en sa chambre fut faict le grant bruit et tonnerre. Ceste chambre pour vray est le corps humain. Car comme l'homme se voit estre beau et fort, incontinent il chiet en peché par lequel la mort luy est preparée. Pour tant Dieu, le voulant de celle mort de l'eternelle misere revocquer, luy fait pres de son corps grant bruict et son par maladie, si que il cuyde mourir. Alors par ce signe plusieurs sont revocquez. Si ce n'estoient les maladies qui nous surviennent, le plus souvent nous demourrions tous en noz pechez: Multiplicate sunt infirmitates eorum postea acceleraverunt. Exemple de sainct Pol, disant: Libenter gloriabor infirmitatibus meis, je me glorifieray en mes infirmitez. Le tiers signe de Cesar fut qu'il eut des lettres testifiant sa mort, lesquelles s'il les eust leues il fust evadé. Dieu nous envoye des lettres pour nous advertir. Ce sont les bonnes inspirations et illustrations de noz consciences, esquelles nous povons lire noz pechez, qui de jour en jour nous blessent et nous veullent occire. Pour tant si nous voulons nostre mort de spiritualité congnoistre, lisons ces lettres et ne les laissons point fermées comme Cesar. Ouvrons noz consciences et voyons qu'il y a dedans si qu'apres la mort du corps ne soyent au jour du jugement ouvertes.

On le voit, l'historicité de César est toute relative. Voici que le fossoyeur de la République se trouve placé, peut-être à la suite d'une mauvaise lecture, dans les premiers temps de la Rome royale. Cette tradition d'une statue de l'empereur détruite par un signe divin concatène en fait deux traditions, celle de la statue de Romulus, et celle qui est mentionnée, chez Pierre le Mangeur, et reprise dans le Speculum Historiale de Vincent de Beauvais :

\footnotetext{
${ }^{12}$ Le texte est ici problématique ; le texte latin propose : Nam si queratur quis sit melior ville vel civitatis, statim preponitur qui plures divitias habet quamvis sit pessimus. Ou le texte latin à disposition était corrompu, ou il a été mal compris par le traducteur.

${ }^{13}$ P. 254-55.
} 


\begin{abstract}
Comestor
Centesimo die ante mortem eius fulmen cecidit iuxta statuam eius in foro, et de nomine eius superscripto .C. capitalem litteram abrupit. Nocte precedenti diem obitus eius, fenestre thalami eius cum tanto strepitu aperte sunt ut exiliens a stratis ruituram domum estimaret. Eadem die cum iret in Capitolium, date sunt ei littere indices mortis imminentis. Que dum referentur occisus in manu eius invente sunt nondum solute. Die sequenti, apparuerunt tres soles in oriente, qui paulatim in unum corpus solare redacti sunt, significantes quod dominium Lucii Antonii, et Marci Antonii, et [A]ugusti in monarchiam rediret. Vel potius quod notitia trini dei et unius toti orbi futura imminebat. ${ }^{14}$
\end{abstract}

J'ignore d'où l'auteur de l'Histoire scolastique tire ses information ${ }^{15}$, mais on ne peut que penser à une contamination du récit de la statue en or de Romulus, qui devait subsister Dum virgo peperit. On sait que cette statue a disparu - preuve s'il en était besoin de l'Incarnation - mais que l'on montrait encore sa place au début du $\mathrm{XVI}^{\mathrm{e}}$ siècle: Palatium Romuli erat apud ecclesiam Sancta Marice Nova absque marmoribus et columnis, cum statua ejus ${ }^{16}$. La mémoire survit donc d'une statue conservée depuis les premiers temps de Rome et qui disparaît au moment de l'Incarnation, et Petrus Comestor montre comment la mort de César finit elle aussi par être liée à une annonce de la Trinité dont l'Incarnation imminebat. César cristallise à lui seul les figures de Romulus et d'Auguste, et le rédacteur des Gesta continue à avoir une grande distance à l'égard des textes antiques, même si le texte latin commence, très scrupuleusement traduit par le Violier, par Legitur in chronicis. Le deuxième élément est celui de cette tempête qui ouvre les fenêtres de la chambre de César. L'épisode est rapporté par Suétone, qui indique ac subito cubiculi fores sponte patuerunt ${ }^{17}$. Le terme de fores est transformé, dès le texte latin des Gesta, en fenestra, manifestement à la suite de Petrus Comestor. Davantage, la chose donne lieu à un commentaire qui souligne le bruit cum tanto strepitu et impetu apertae sunt, ut domum ruituram aestimaret. Le dernier élément est celui de ces lettres données à César et non décachetées, qui l'avertissaient du complot, élément présent de même chez Suétone.

Cette suite est exemplaire de la capacité de l'auteur des Gesta à s'approprier tout type d'élément merveilleux pour le recombiner selon ses besoins. On pourra

14 Vincent de Beauvais, Speculum Historiale,lib. VII, cap. XLI; texte repris du site de l'ARTEM. Un texte légèrement fautif se trouve dans l'édition de Douai, qui propose Cliteram au lieu de . C. capitalem litteram (Lib VI, cap. 41, p. 187).

${ }^{15}$ On peut proposer une réminiscence de Suétone, XCV Post necem Caesaris reuerso ab Apollonia et ingrediente eo urbem repente liquido ac puro sereno circulus ad speciem caelestis arcus orbem solis ambiit ac subinde Iuliae Caesaris filiae monimentum fulmine ictum est. Mais il s'agit ici de la fille de Jules César, et il n'est pas question d'effacer une lettre.

${ }^{16}$ Francesco Albertini, Mirabilia Rome, editum Francisco Albertino Florentino, Romani Morin, Lyon, 1520, f $19 \mathrm{v}^{\circ}$. Cf. Également In Romuliano palatio sunt due edes, Pietatis et Concordie, ubi posuit Romulus statua suam auream, dicens: "Non cadet, donec virgo pariat". Statim ut virgo peperit, illa corruit..., in Cesare D'Onofrio, Visitiamo Roma mille anni fa. La città dei Mirabilia (Roma 1988), ch. 5, disponible sur internet.

${ }^{17}$ Suétone, Vies des douze Césars, éd. H. Ailloud, Paris, Belles Lettres, 1954, t. I, p. 57. 
noter que des traits tout aussi étonnants rapportés par Suétone sont écartés, la tombe dont la découverte annonce la mort d'un membre de la gens Julia, les chevaux qui pleurent $^{18}$ : il est bien question de forger un exemplum autour d'épisodes choisis dans un dessein précis. Ici, on importe purement et simplement un épisode étranger à la vie de César, on adapte l'autre, on rapporte le dernier sans avoir besoin de le transformer. L'objet de la prédication est cohérent, bien que forgé de pièces et de morceaux. Mais d'autres traits apparaissent, qui soulignent l'originalité de la figure de César. Après avoir été un avatar de la figure divine, une représentation exemplaire du législateur, le voici maintenant figure de l'homme, refusant d'entendre les messages qui lui sont envoyés. Si le prince est Dieu, il est aussi chacun de nous, et les miroirs des princes, comme genre littéraire et moral, dépassent largement le public curial pour s'adresser à chacun.

Les signes et prodiges qui s'associent à César vont désormais constituer des éléments caractéristiques du personnage, entouré d'éléments surnaturels dont il ne tient pas compte. Mieux, il semble que leur emploi soit comme emblématique du traitement qu'il reçoit : son nom est comme effacé - tout au moins la première lettre, le bruit qui le réveille lui reste inintelligible, il ne lit pas les lettres qui lui sont envoyées. Ainsi le personnage historique est-il comme instrumentalisé à des fins apologétiques, indépendamment de la réalité historique. Mais ce qui semble dominer ce récit, c'est une forme presque donjuanesque de héros, indifférent aux signes du destin et mu par une volonté aveugle. En ceci, César devient un personnage exemplaire, car son excès même permet de mettre en scène la nécessité, pour chacun de nous qui sommes soumis à l'aurea mediocritas, de prendre garde aux signes du destin. L'effacement du nom devient ainsi une sorte de châtiment anticipé, dont le Moyen Âge saura être l'illustration, lui qui semble méconnaître avec persévérance la figure de l'écrivain comme celle du général.

Un dernier texte sera exemplaire de cette démarche : la reprise du passage du Rubicon va donner lieu à une récriture exceptionnelle, dont on va pouvoir suivre les jalons, de Suétone et Lucain aux versions médiévales. Commençons par le texte de Suétone :

«Etiam nunc», inquit, « regredi possumus ; quod si ponticulum transierimus, omnia armis agenda erunt. »

XXXII. Cunctanti ostentum tale factum est. Quidam eximia magnitudine et forma in proximo sedens repente apparuit harundine canens; ad quem audiendum cum praeter pastores plurimi etiam ex stationibus milites concurrissent interque eos et aeneatores, rapta ab uno tuba prosiliuit ad flumen et ingenti spiritu classicum exorsus pertendit ad alteram ripam. Tunc Caesar : "Eatur», inquit, " quo deorum ostenta et inimicorum iniquitas uocat. Jacta alea est ", inquit. ${ }^{19}$

${ }^{18}$ Ibid.

19 «Maintenant, nous pouvons encore revenir en arrière, mais une fois que nous aurons franchi ce peit point, tout devra être réglé par les armes.

XXXII. Comme il hésitait, il reçut un signe d'en haut. Un homme d'une taille et d'une beauté extraordinaires apparut soudain, assis tout près de là et jouant du chalumeau; des bergers étant accourus pour l'entendre ainsi qu'une foule de soldats des postes voisins, et parmi eux également des trompettes, cet homme prit à l'un d'entre eux son instrument, s'élança vers la 
Au moment où hésite César devant le pas à franchir, un prodige - ostentum lui montre la voie, prodige qui se manifeste à la fois par sa taille inhabituelle et sa beauté, et par la beauté de la musique qu'il joue, au point d'attirer à la fois pasteurs joueurs de flageolet et de musette - et soldats - les cuivres aeneatores. C'est à la fois la guerre et la paix qui concourent ici, comme mimant l'enjeu de la décision à prendre par César au sein de ce qui va être une guerre civile. La figure gigantesquequidam - apparaît de façon inexpliquée, comme une émanation de la terre même. Quant à César, doublé dans sa décision de traverser par cette figure qui ravit à un soldat la «trompette guerrière»-rapta ab uno tuba-il se contente d'accepter passivement le signe, avec la forme passive du verbe ire. Dans ces lignes où je rappelle des choses bien connues, c'est un signe qui lui est envoyé et le général est comme dépossédé de sa décision ${ }^{20}$. Il en est tout autrement dans le passage correspondant du livre I de la Pharsale :

\author{
iam gelidas Caesar cursu superaverat Alpes \\ ingentisque animo motus bellumque futurum \\ ceperat. ut ventum est parvi Rubiconis ad undas, \\ ingens visa duci patriae trepidantis imago \\ clara per obscuram voltu maestissima noctem \\ turrigero canos effundens vertice crines \\ caesarie lacera nudisque adstare lacertis \\ et gemitu permixta loqui: "quo tenditis ultra? \\ quo fertis mea signa, viri? si iure venitis, \\ si cives, huc usque licet. » tum perculit horror \\ membra ducis, riguere comae gressumque coercens \\ languor in extrema tenuit vestigia ripa. \\ mox ait " o magnae qui moenia prospicis urbis \\ Tarpeia de rupe Tonans Phrygiique penates \\ gentis Iuleae et rapti secreta Quirini \\ et residens celsa Latiaris Iuppiter Alba \\ Vestalesque foci summique o numinis instar \\ Roma, fave coeptis. non te furialibus armis \\ persequor: en, adsum victor terraque marique \\ Caesar, ubique tuus (liceat modo, nunc quoque) miles. \\ ille erit ille nocens, qui me tibi fecerit hostem. " \\ inde moras solvit belli tumidumque per amnem \\ signa tulit propere: ... ${ }^{21}$
}

rivière et, sonnant la marche avec une puissance formidable, passa sur l'autre rive. Alors César dit: «Allons où nous appellent les signes des dieux et l'injustice de nos ennemis. Le sort en est jeté. » Suétone, Id., p. 23.

${ }_{21}^{20}$ Merci à Monique Bouquet pour les échanges fructueux autour de ce texte.

21 Lucani bellum civile, I, v. 183-205, ed. A. E. Housman, Oxford 1926. Le texte est disponible sur le site de la Bibliotheca Augustana. «Déjà César avait franchi le sommet glacé des Alpes, l'esprit violemment agité, le cœur plein de la guerre future. À peine fut-il arrivé aux bords étroits du Rubicon, une grande ombre lui apparut : c'était l'image de la patrie ! Elle brillait dans l'ombre de la nuit. Elle était tremblante et consternée. De son front couronné de 
Si une figure apparaît, c'est celle de la République visa duci patriae trepidantis imago, sous les figures d'une victime suppliante et échevelée. César est cette fois-ci considéré comme le chef de guerre ayant déjà prémédité sa rébellion ingentisque animo motus bellumque futurus. Devant le spectacle de la Patrie souffrante, il lui faut réfléchir à une réponse - il n'est pas si fréquent que le monosyllabe mox soit mis en anacrouse du vers et de la phrase, comme pour souligner le délai de réflexion qui l'amène à cette réponse rhétorique et habile ille erit ille nocens, qui me tibi fecerit hostem. Nulle indécision, nulle passivité dans les gestes de César; il est l'image de l'homme mû par des passions que la rhétorique se chargera de justifier. Lucain, par ailleurs, joue avec un sens aigu du spectaculaire sur l'apparition, qui gagne en pathétique ce qu'elle perd en merveilleux. L'épisode rapporté par Suétone pouvait passer pour authentique, exemple intéressant du «merveilleux objectif» que peut parfois proposer l'histoire. Ce que propose Lucain relève explicitement de l'ornatio littéraire, et perd toute validité historique.

Ce détour par les textes latins permet de voir dans quelle tradition s'inscrit le texte du Violier. Héritier de Lucain, il fabrique un récit composite, où apparaissent non seulement César et le Rubicon, mais une sorte de mythe fondateur de la guerre civile.

19 De peché d'orgueil

On lit es gestes rommaines qu'il y avoit jadis ung prince des Rommains nommé Pompée qui estoit associé de la fille de Cesar par droit de mariage. Ces deux seigneurs convindrent en ung conseil ensemble, tellement qu'il estoit conclu entre les deux que tout le domaine du monde seroit à eulx mis et adjousté à l'empire. Le cas fut que Pompée transmist Cesar à combatre diverses regions. Pour ce qu'il estoit jeusne, pour tant le vouloit au labeur apprendre. Pompée voulut demourer à Romme gardant les regions, et si donna à Cesar terme dedans cinq ans, laquelle chose s'il ne la faisoit, privé devoit estre de son droict perpetuellement. Cesar assembla grande exercite, cheminant es parties à luy ordonnées, esquelles il trouva gens fors belliqueulx, lesquelz il ne peult pas en si peu de temps superer et surmonter, mieulx aymant Pompée lors offenser que laisser la bataille. Parquoy Pompée l'aliena de son droict et luy deffendit la cité de Rome, tellement qu'il estoit sans oser s'approcher, comme interdict de Pompée. Quant la bataille de Cesar fut finie, ledit Cesar voulut retourner à Romme. Pour y venir secretement, il luy faillut passer par le fleuve

tours, ses cheveux blancs tombaient épars. Debout devant lui, les bras nus, elle prononce ces paroles entrecoupées de gémissements: «Où allez-vous, soldats, où portez-vous mes enseignes? Si vous respectez les lois, si vous êtes citoyens, arrêtez! Un pas de plus serait un crime. » À ces mots, le cœur de César est saisi d'horreur; ses cheveux se dressent sur sa tête, et la langueur dont il est abattu enchaîne ses pas au rivage. Mais bientôt : «O Jupiter ! s'écriat-il, ô toi ! que mes aïeux ont adoré dans Albe naissante, et qui, du haut du Capitole, veilles aujourd'hui sur la reine du monde; et vous, dieux tutélaires des Troyens, qu'Énée apporta dans l'Ausonie; et toi, Romulus, qui, enlevé au ciel, devins l'objet de notre culte; et toi, Vesta, qui vois sur tes autels brûler sans cesse le feu sacré ; et toi, Rome, qui fus toujours une divinité pour moi, favorisez mon entreprise. Non, Rome, ne crois pas voir César te poursuivre, armé du flambeau des Furies. Vainqueur sur la terre et sur les mers, il est encore à toi, si tu le veux; il est ton soldat, il le sera partout. Celui-là seul sera criminel qui fera de César l'ennemi de Rome ». À ces mots, sans plus différer, il fit passer le fleuve à ses troupes. 
Rubicon et là luy apparut ung ymage sus l'eaue grant à merveilles, lequel parla du meillieu de l'eaue et dist: O Cesar, si tu viens pour la rommaine prosperité, il t'est parmis venir jusques icy. Sinon, ne presume plus avant passer et venir sus ce fleuve. Lors dist Cesar:

- J'ay tousjours pour le prouffit rommain traveillé et suis encores prestz à souffrir les labeurs pour la chose publicque multiplier, et les dieux lesquelz tous jours adore m'en soient tesmoings.

Cela dit, l'ymage disparut. Apres cela incontinent Cesar frappa son dextrier, passa le fleuve. Puis dist au contraire de ses premieres paroles qu'il relinquoit et laissoit les droictz rommains et que jamais ne cesseroit de persecuter son sire Pompée. Cela fist. Car de ce jour en avant s'esforça de le destruire!

L'exposition moralle sus l'histoire devant dicte

Par cestuy Pompée qui estoit ancien, entendons Dieu le createur de tous, qui a esté des le commencement et tousjours sera. Par Cesar entendons Adam qui fut prince de tous les hommes, duquel la fille fut à Dieu par foy espousée est l'ame. Dieu doncques, voulant prouver Adam, comme Pompée Cesar, le mist en paradis terrestre pour le culturer et garder, lequel tout incontinent de son estat si digne se glorifiant et voulant à sa femme plaire pour obeyr au dyable viola ung seul commandement que Dieu luy avoit baillé. Pour la cause Dieu le chassa de paradis non seullement terrestre mais aussi de l'empire celeste. Toutesfois Adam, sperant en son animé courage ce qu'il avoit perdu et obmis recuperer, tant qu'il peust continuellement laboura. Mais il ne povoit toutesfois en son predict estat revenir jusques à l'advenement de nostre seigneur Jesucrist, le vray ymage de Dieu son pere, qui sus les eaulx de nostre redemption apparut à son baptesme, qui nous dist à tous voulant retourner en gloire: Nisi quis regnatus fuerit ex aqua et spiritu sancto, etc. Qui ne sera regeneré de l'eaue, et du Sainct Esperit ne peult aller en paradis, la cité capitalle d'amour et de joye. Plusieurs sont qui viennent à ce fleuve promettant assez devant les tesmoings par procureurs, qui sont leurs parrins, servir à Dieu, et virilement contre le dyable combatre pour la cité de paradis, comme Cesar pour la cité de Romme. Mais quant ilz ont fait leur veu de leur promesse, s'oblient et contempnent les commandemens de Dieu, comme fist Absalon apres que il fut à son pere reconcilié. Dieu nous garde de faire selon cest exemple.

On sait en effet que l'alliance du Triumvirat a été scellée par le mariage de Pompée et de Julia, fille de César. Dans la version qu'en donne le Violier, Crassus a complètement disparu, et le monde est partagé entre les deux généraux, comme le pouvoir. Malgré le motif du mariage de la fille de César, le récit semble reprendre la trame thébaine d'un partage de la royauté : Pompée et César ne sont ici que des avatars d'Étéocle et Polynice, et ce d'autant plus qu'en 52, au moment de notre récit, Julia comme Crassus sont morts et laissent les deux protagonistes en présence. La traduction du Violier est moins claire que le texte latin, qui parle explicitement des plagas que doit difficilement combattre César à l'extérieur - il s'agit de la révolte gauloise - alors que Pompée se réserve une police intérieure sans difficultés semble-t-il. Au bout de cinq ans, l'échange prévu des pouvoirs ne peut avoir lieu, puisque César n'a pas encore accompli sa tâche «mieulx aymant Pompée lors offenser que laisser la bataille, parquoy Pompée l'aliena de son droit et luy deffendit

${ }^{22}$ Éd. Hope, p. $75-77$. 
la cité de Rome, tellement qu'il estoit sans oser s'approcher, comme interdict de Pompée ». Le premier jugement porté, implicitement, sur César, est donc celui d'un fidèle serviteur, respectueux des lois, et d'une certaine façon injustement relégué hors d'un royaume qu'il a contribué à défendre. Il n'empêche cependant que le désir de posséder le monde entier répartit sur eux deux la même faute de démesure.

La figure qui interpelle César n'est pas manifestement issue de Lucain; certes elle parle, comme le poète. Mais au lieu d'apparaître dans l'ombre de la nuit (clara per obscuram voltu maestissima noctem), c'est sur l'eau qu'elle surgit. Elle n'est pas identifiée comme la Patrie : on dit simplement d'elle qu'elle est «grant à merveilles »- le texte latin propose imago magna: une telle formule nous rapproche de Suétone, mais avec des mots différents. De même, le discours, s'il reprend Lucain pour l'ensemble, le gauchit; la Patrie demandait chez Lucain si César et ses troupes venaient en citoyens soumis aux lois si iure venitis,/ si cives, la question est ici de venir pour le bien de Rome, pour sa prospérité : malgré l'essor du droit romain, le droit et la loi restent secondaires dans l'Europe médiévale, ou tout au moins associés à la richesse des habitants, l'engagement pris par César de venir pour «pour le prouffit rommain » et «pour la chose publicque» le montre. C'est l'interdit de Pompée qui amène César à se rebeller après la traversée du cours d'eau, conformément à ce que propose la Pharsale :

Hic, ait, hic pacem temerataque iura relinquo;

Te, Fortuna, sequor. Procul hinc iam foedera sunto. Credidimus fatis. Utendum est iudice bello. ${ }^{23}$

La figure, monitoire et univoque chez Suétone ou même chez Lucain est ici plus ambiguë, puisqu'elle se pose en gardienne du passage et se volatilise non pas la décision prise, mais l'engagement solennellement formulé, comme si celui-ci seul autorisait le franchissement du Rubicon. Le général, victime d'une règle injuste et d'un sentiment de devoir à l'égard de sa patrie plus grand même que celui qu'il doit à son gendre, passe à cheval, pour revenir sur sa parole et prononcer son double défi à l'égard des lois de Rome et de Pompée.

Ce simple élément permet de démonter le système symbolique mis en place par le prédicateur qui a compilé ce récit. Jusqu'ici le Rubicon se traversait à pied, comme le montre le texte de Suétone. S'il importe que César soit à cheval et chevauche pour traverses le fleuve, reniant aussitôt ses engagements, c'est qu'il est dès ce moment figure de la démesure, capable de revenir sur sa parole.

C'est la moralisation qui contient, évidemment, les clefs de la modification du récit. Elle est cependant étonnante, en ce que la Gaule rebelle semble difficilement assimilable au jardin d'Éden; toute se passe pourtant comme si la pleine possession des terres offertes pas Pompée était perdue par la faute de César, comme s'il était obligé d'expier par ses guerres gauloises une faute antérieure: «Dieu doncques, voulant prouver Adam, comme Pompée Cesar, le mist en paradis terrestre pour le culturer et garder, lequel tout incontinent de son estat si digne se glorifiant et voulant à sa femme plaire pour obeyr au dyable viola ung seul commandement que Dieu luy avoit baillé ». S'il y a épouse, c'est celle de Pompée, la

${ }^{23}$ Op. cit, I, v 225-227. 
fille de César et pas le contraire : on voit que dans la procédure de moralisation, souvent très rigoureuse, tous ces préliminaires imprécis n'ont pas grande valeur; ils ont pour seule fonction de mettre en place une relation de parenté assez floue, une faute, une transgression: celle-ci entraîne une sorte d'expiation, qui semble s'achever lors du passage du Rubicon, perçu comme une métaphore baptismale c'est un classique de la littérature de moralisation de le voir dans toute traversée de fleuve. Jules César figure indifféremment alors l'Homme et le Christ, en même temps que l'apparition est: «à l'advenement de nostre seigneur Jesucrist, le vray ymage de Dieu son pere, qui sus les eaulx de nostre redemption apparut à son baptesme». Si le Rubicon est baptismal, il importe que Rome soit à son tour une figure de la Jérusalem céleste, ce que confirme le texte: «Qui ne sera regeneré de l'eaue, et du Sainct Esperit ne peult aller en paradis, la cité capitalle d'amour et de joye $»$.

Il ne reste plus qu'à montrer comment, reniant ses engagements, César est une figure négative, maléfique presque, qui va plonger l'Italie à feu et à sang, contribuer à la mort de Pompée et s'emparer de Rome : dans le système de lecture de la moralisation, cela reviendrait à tuer Dieu et à conquérir, armes à la main, la Jérusalem céleste.

On le voit, le système de moralisation proposé par le Violier, à la suite des Gesta, est bien fragile et paradoxal. On pourrait conclure aisément sur la médiocrité du texte ; c'est oublier le nombre de manuscrits qui nous le conservent, et même le relatif succès, sous sa forme imprimée, de la traduction française. Même si l'on peut penser que d'autres histoires, mieux menées, ont fait la force de ce recueil, il convient de rappeler que la version française en a éliminé un certain nombre, et que cette histoire a été épargnée. Certes, elle a basculé du chapitre XIII des Gesta au chapitre XIX du Violier: l'histoire de Jules César se trouve après celle de Julien l'Hospitalier (ch. XVIII), comme pour constituer un contre-exemple, et montrer comment, autour d'une idée de parricide - il s'agit bien de tuer Pompée comme Julien tue son propre père - deux modèles s'opposent, dont un qu'il faut éviter : «Dieu nous garde de faire selon cest exemple».

Jules César devient ainsi, dès qu'il est abordé en tant que personne, une figure globalement négative, puisqu'incapable d'écouter les signes divins, qu'il s'agisse des prodiges annonçant sa mort ou des questions solennellement posées par des figures surnaturelles. Tant qu'il est abstraitement représenté, il peut endosser les responsabilités de l'empereur, gardien du droit et de la justice; dès qu'il est question de lui en tant qu'être humain, c'est l'orgueil qui domine, orgueil qui devient en quelque sorte emblématique de tout pécheur, modèle à ne pas suivre, toujours sourd et aveugle aux injonctions et aux signes du destin, toujours suspect de duplicité et coupable de démesure; c'est ainsi une sorte de fatalité qui caractérise son cheminement, fatalité qui le montre à la fois exemplaire et bien proche de chacun de nous, comme le souligne le commentaire qui parle du nombre des baptisés incapables de tenir leurs engagements.

«Un»César est ainsi fabriqué, comme n'importe quelle figure historique, pour répondre aux attentes et aux interrogations d'une époque donnée. Beaucoup moins docile que Virgile, sa stature militaire et politique reste, de quelque façon que l'aborde le Moyen Âge religieux, irréductible aux canons de compréhension du 
christianisme. C'est peut-être cela que paie César en devenant une figure emblématique de l'orgueil et de la démesure.

Il n'empêche; si Lucain a pu proposer du général romain un portrait assez défavorable pour que pendant des siècles ce soit lui qui incarne la série des vices et des tares que dénonce le Violier, on voit, par la maladresse de la moralisation, combien cette figure est proprement irréductible à l'univers de pensée médiéval : Jules César ne peut se réduire à un récit de baptême ou à une apologie de l'humilité, il est à la fois juste, habile et politique, modèle de vertus laïques anachroniques, sous-employé dans les mentalités religieuses de la fin du Moyen Âge, et qui trouvera sa place à la Renaissance, au moment de la redécouverte de l'Antiquité et de l'apogée des condottiere italiens.

Denis Hüe,

CETM-CELAM,

Université Rennes 2 Haute Bretagne 